Bull. Korean Math. Soc. 51 (2014), No. 3, pp. 659-666

http://dx.doi.org/10.4134/BKMS.2014.51.3.659

\title{
$T$-NEIGHBORHOODS IN VARIOUS CLASSES OF ANALYTIC FUNCTIONS
}

\author{
Saeid Shams, Ali Ebadian, Mahta Sayadiazar, and Janusz Sokó
}

AbSTRACT. Let $\mathcal{A}$ be the class of analytic functions $f$ in the open unit disk $\mathbb{U}=\{z:|z|<1\}$ with the normalization conditions $f(0)=f^{\prime}(0)-1=0$. If $f(z)=z+\sum_{n=2}^{\infty} a_{n} z^{n}$ and $\delta>0$ are given, then the $T_{\delta}$-neighborhood of the function $f$ is defined as

$$
T N_{\delta}(f)=\left\{g(z)=z+\sum_{n=2}^{\infty} b_{n} z^{n} \in \mathcal{A}: \sum_{n=2}^{\infty} T_{n}\left|a_{n}-b_{n}\right| \leq \delta\right\},
$$

where $T=\left\{T_{n}\right\}_{n=2}^{\infty}$ is a sequence of positive numbers. In the present paper we investigate some problems concerning $T_{\delta}$-neighborhoods of functions in various classes of analytic functions with $T=\left\{2^{-n} / n^{2}\right\}_{n=2}^{\infty}$. We also find bounds for $\delta_{T}^{*}(A, B)$ defined by

$$
\delta_{T}^{*}(A, B)=\inf \left\{\delta>0: B \subset T N_{\delta}(f) \text { for all } f \in A\right\},
$$

where $A, B$ are given subsets of $\mathcal{A}$.

\section{Introduction}

Let $\mathcal{A}$ denote the class of analytic functions $f$ in the open unit disk $\mathbb{U}=$ $\{z:|z|<1\}$ with the normalization conditions $f(0)=f^{\prime}(0)-1=0$. If $f(z)=z+\sum_{n=2}^{\infty} a_{n} z^{n}$, then the $T_{\delta}$-neighborhood of the function $f$ is defined as

$$
T N_{\delta}(f)=\left\{g(z)=z+\sum_{n=2}^{\infty} b_{n} z^{n} \in \mathcal{A}: \sum_{n=2}^{\infty} T_{n}\left|a_{n}-b_{n}\right| \leq \delta\right\},
$$

where $\delta$ is a positive number and $T=\left\{T_{n}\right\}_{n=2}^{\infty}$ is a sequence of positive numbers. St. Ruscheweyh in [14] considered $T=\{n\}_{n=2}^{\infty}$ and showed that if $f \in \mathcal{C}$, then $T N_{1 / 4}(f) \subset \mathcal{S}^{*}$, where $\mathcal{C}, \mathcal{S}^{*}$ denote the well known classes of convex and starlike functions, respectively. In $[4,5,6,7,10,11,12,17,18]$ other authors investigated some interesting results concerning neighborhoods of several classes of analytic functions. Some of the relations between the neighborhoods for a certain class of analytic functions was described by S. Shams et al. [15].

Received January 18, 2013; Revised July 10, 2013

2010 Mathematics Subject Classification. Primary 30C45; Secondary 30C50, 40 A05.

Key words and phrases. analytic functions, univalent, starlike, convex, close-to-convex, concave functions, neighborhood, $T_{\delta}$-neighborhood, $T$-factor 
Also U. Bednarz and J. Sokół in [7] considered $T=\left\{\frac{1}{n^{2}(n-1)}\right\}_{n=2}^{\infty}$ and investigated $T_{\delta}$-neighborhood for various subclasses of analytic functions. Motivated by the above results, we consider in this paper $T_{\delta}$-neighborhood (1.1) with $T=\left\{2^{-n} n^{-2}\right\}_{n=2}^{\infty}$. We use this sequence because it is sufficiently strongly convergent to 0 , which is necessary for the series considered here to be convergent. Notice that $\sum_{n=1}^{\infty} 2^{-n} n^{-2}=\pi^{2} / 12-(\log 2)^{2} / 2$ and it is the value of dilogarithm at $1 / 2,[13]$.

The convolution or Hadamard product of the functions $f$ and $g$ of the form

$$
f(z)=z+\sum_{n=2}^{\infty} a_{n} z^{n}, \quad g(z)=z+\sum_{n=2}^{\infty} b_{n} z^{n}, \quad|z|<1,
$$

is defined by

$$
(f * g)(z)=z+\sum_{n=2}^{\infty} a_{n} b_{n} z^{n} .
$$

Definition $1.1([2])$. Let us consider the functions $f$ that are meromorphic and univalent in $\mathbb{U}$, holomorphic at 0 and have the expansion $f(z)=z+\sum_{n=2}^{\infty} a_{n} z^{n}$. If, in addition, the complement of $f(\mathbb{U})$ with respect to $\mathbb{C}$ is convex, then $f$ is called a concave univalent function. The class of all concave functions is denoted by $\mathcal{C}$ o.

It is well known [1], that if $f \in \mathcal{C} o$, then $\left|a_{n}\right| \geq 1$ for all $n>1$ and equality holds if and only if $f(z)=z /(1-\mu z),|\mu|=1$ (see [1,3]). The authors in [2] considered the class $\mathcal{C} o(p) \subset \mathcal{C} o$ consisting of all concave functions that have a pole at the point $p$ and are analytic in $|z|<|p|$. They proved that if $f \in \operatorname{Co}(1)$, then

$$
\left|a_{n}-\frac{n+1}{2}\right| \leq \frac{n-1}{2} \text { for } n \geq 2,
$$

and equality holds only for the function $f_{\theta}$ defined by

$$
f_{\theta}(z)=\frac{2 z-\left(1-e^{i \theta}\right) z^{2}}{2(1-z)^{2}}, \quad|z|<1 .
$$

It is well known that if $f \in \mathcal{C} o(1)$, then the complement of $f(\mathbb{U})$ can be represented as the union of a set of mutually disjoint half-lines (the end point of one half-line can lie on the another half-line), so $f(\mathbb{U})$ is a linearly accessible domain in the strict sense (see $[8,16])$.

The authors in [7] also showed that $\mathcal{C} o(1) \subset \mathcal{K}$, where $\mathcal{K}$ is the set of closeto-convex functions.

\section{Main results}

Throughout this section $T$ will always be the sequence given by

$$
T=\left\{T_{n}\right\}_{n=2}^{\infty}=\left\{2^{-n} n^{-2}\right\}_{n=2}^{\infty},
$$

unless otherwise stated. 
Theorem 2.1. If $f, g \in \mathcal{A}$ are of the form $f(z)=z+\sum_{n=2}^{\infty} a_{n} z^{n}, g(z)=$ $z+\sum_{n=2}^{\infty} b_{n} z^{n}$ with $\left|a_{n}\right| \leq n$ and $\left|b_{n}\right| \leq n$ for $n=2,3,4, \ldots$, then $g \in$ $T N_{\log \{4 / e\}}(f)$, where $T$ is given in (2.1). The number $\log \{4 / e\}$ is the best possible.

Proof. A simple calculation shows that

$$
\sum_{n=1}^{\infty} \frac{z^{n}}{n 2^{n}}=\int_{0}^{z} \sum_{n=1}^{\infty} \frac{\zeta^{n-1}}{2^{n}} \mathrm{~d} \zeta=\int_{0}^{z} \frac{1 / 2}{1-\zeta / 2} \mathrm{~d} \zeta=\log \frac{1}{1-z / 2}, \quad|z|<2
$$

so we have

$$
\sum_{n=1}^{\infty} \frac{1}{n 2^{n}}=\log 2
$$

and then

$$
\sum_{n=2}^{\infty} T_{n}\left|a_{n}-b_{n}\right| \leq \sum_{n=2}^{\infty} \frac{2 n}{n^{2} 2^{n}}=2 \sum_{n=2}^{\infty} \frac{1}{n 2^{n}}=2 \log 2-1=\log \{4 / e\} .
$$

For the functions

$$
f(z)=z+\sum_{n=2}^{\infty} a_{n} z^{n}=z+\sum_{n=2}^{\infty} n z^{n}, \quad g(z)=z+\sum_{n=2}^{\infty} b_{n} z^{n}=z-\sum_{n=2}^{\infty} n z^{n}
$$

we have

$$
\sum_{n=2}^{\infty} T_{n}\left|a_{n}-b_{n}\right|=2 \sum_{n=2}^{\infty} \frac{1}{n 2^{n}}=\log \{4 / e\} .
$$

Therefore, the number $\log \{4 / e\}$ cannot be replaced by a smaller one and it is the best possible.

It is well known that $\mathcal{C} \subset \mathcal{S}^{*} \subset \mathcal{K} \subset \mathcal{S}$ (see [9]), where $\mathcal{S}, \mathcal{S}^{*}, \mathcal{C}$ and $\mathcal{K}$ denote the classes of univalent, starlike, convex and close-to-convex functions, respectively. Also, if $f \in \mathcal{S}^{*}$, then $\left|a_{n}\right| \leq n, n=2,3, \ldots$, while if $f \in \mathcal{C}$, then $\left|a_{n}\right| \leq 1, n=2,3, \ldots$.

Therefore we obtain the following corollary.

Corollary 2.2. If $f \in \mathcal{S}$, then we have

$$
\mathcal{S} \subset T N_{\log \{4 / e\}}(f),
$$

where $T$ is given in (2.1).

The constant $\log \{4 / e\} \approx 0.386$ seems not to be the best possible. An interesting open problem is to find the smallest constant $\varrho$ such that for each $f \in \mathcal{S}$

$$
\mathcal{S} \subset T N_{\varrho}(f),
$$


where $T$ is given in (2.1). For the Koebe function $f(z)=z /(1-z)^{2}$ and $g(z)=-f(-z)$ we have $f, g \in \mathcal{S}$ and

$f(z)=z+\sum_{n=2}^{\infty} a_{n} z^{n}=z+\sum_{n=2}^{\infty} n z^{n}, g(z)=z+\sum_{n=2}^{\infty} b_{n} z^{n}=z+\sum_{n=2}^{\infty}(-1)^{n-1} n z^{n}$

so by $(2.2)$

$$
\sum_{n=2}^{\infty} T_{n}\left|a_{n}-b_{n}\right|=\sum_{k=1}^{\infty} \frac{4 k}{(2 k)^{2} 2^{2 k}}=\log \{4 / 3\} .
$$

Therefore, the number $\varrho$ cannot be smaller than $\log \{4 / 3\}$. We conjecture that $\varrho=\log \{4 / 3\}=0.28768 \cdots$.

Corollary 2.3. Let $f \in \mathcal{C}$. Then $\mathcal{S} \subset T N_{\beta}(f)$ with

$$
\beta=\log \{2 / e\}+\frac{\pi^{2}}{12}-\frac{(\log 2)^{2}}{2}=0.275 \cdots .
$$

Proof. At first, note that

$$
f_{2}(x)=-\int_{1}^{x} \frac{\log t}{t-1} \mathrm{~d} t, \quad x \in[0,2],
$$

is the dilogarithm. From the tables of dilogarithms we have

$$
\begin{aligned}
& f_{2}(x)=\sum_{k=1}^{\infty}(-1)^{k} \frac{(x-1)^{k}}{k^{2}}, \quad x \in[0,2], \\
& f_{2}(x)+f_{2}(1-x)=-\log \{x\} \cdot \log \{1-x\}+\pi^{2} / 6, \\
& f_{2}(1+x)-f_{2}(x)=-\log \{x\} \cdot \log \{x+1\}-\pi^{2} / 12-f_{2}\left(x^{2}\right) / 2 .
\end{aligned}
$$

Therefore, using (2.5) and (2.6) we obtain

$$
\sum_{n=1}^{\infty} \frac{1}{n^{2} 2^{n}}=f_{2}(1 / 2)=\frac{\pi^{2}}{12}-\frac{(\log 2)^{2}}{2} .
$$

If

$$
f(z)=z+\sum_{n=2}^{\infty} a_{n} z^{n} \in \mathcal{C}, \quad g(z)=z+\sum_{n=2}^{\infty} b_{n} z^{n} \in \mathcal{S},
$$

then $\left|a_{n}\right| \leq 1,\left|b_{n}\right| \leq n$ and by (2.3), (2.8) we have

$$
\begin{aligned}
\sum_{n=2}^{\infty} T_{n}\left|a_{n}-b_{n}\right| \leq \sum_{n=2}^{\infty} \frac{n+1}{n^{2} 2^{n}} & =\sum_{n=2}^{\infty} \frac{1}{n 2^{n}}+\sum_{n=2}^{\infty} \frac{1}{n^{2} 2^{n}}=\log \{2 / e\}+f_{2}(1 / 2) \\
& =0.275 \cdots .
\end{aligned}
$$

In a similar way as in Corollary 2.2 , the constant $0.275 \cdots$ given in Corollary 2.3 is also not sharp but if the class $\mathcal{S}$ is replaced by the much larger class of all normalized analytic functions $f$ such that $\left|a_{n}(f)\right| \leq n$ for $n \geq 2$, then (2.4) 
becomes sharp. The best possible constant in the case $f \in \mathcal{S}$ is not known. We conjecture that the sharp constant is attained by the functions

$$
f(z)=z+\sum_{n=2}^{\infty} a_{n} z^{n}=\frac{z}{(1-z)^{n}} \quad g(z)=z+\sum_{n=2}^{\infty} b_{n} z^{n}=\frac{z}{1+z} .
$$

It is clear that $f \in \mathcal{S}$ and $g \in \mathcal{C}$. Moreover,

$$
\begin{aligned}
\sum_{n=2}^{\infty} T_{n}\left|a_{n}-b_{n}\right| & =\sum_{n=2}^{\infty} \frac{n+1}{2^{n} n^{2}}-\sum_{n=2}^{\infty} \frac{1+(-1)^{n-1}}{2^{n} n^{2}} \\
& =\log \{2 / e\}+f_{2}(1 / 2)-\sum_{k=1}^{\infty} \frac{2}{2^{2 k+1}(2 k+1)^{2}}
\end{aligned}
$$

From the tables of dilogarithms we have

$$
\sum_{k=1}^{\infty} \frac{2}{2^{2 k+1}(2 k+1)^{2}}=\int_{0}^{1 / 2} \frac{1}{t} \log \frac{1+t}{1-t} \mathrm{~d} t-1=f_{2}(1 / 2)-f_{2}(3 / 2)-1 .
$$

By (2.7) we have

$$
f_{2}(1 / 2)-f_{2}(3 / 2)=\frac{f_{2}(1 / 4)}{2}+\frac{\pi^{2}}{12}-\log \{2\} \cdot \log \{3 / 2\} .
$$

Applying this in (2.9) we further get,

$$
\begin{aligned}
& \sum_{n=2}^{\infty} T_{n}\left|a_{n}-b_{n}\right| \\
= & \log \{2 / e\}+f_{2}(1 / 2)-\left\{\frac{f_{2}(1 / 4)}{2}+\frac{\pi^{2}}{12}-\log \{2\} \cdot \log \{3 / 2\}-1\right\} \\
= & \log \{2\} \cdot \log \{3 e /(2 \sqrt{2})\}-\frac{f_{2}(1 / 4)}{2}=0.24473 \cdots,
\end{aligned}
$$

because $f_{2}(1 / 4)=0.978469393 \cdots$. Therefore, the smallest constant $\beta$ such that $\mathcal{S} \subset T N_{\beta}(f)$ for each $f \in \mathcal{C}$ lies between $0.2447 \cdots$ and $0.275 \cdots$. We conjecture that it is the first number.

Theorem 2.4. Let $f, g_{1}, g_{2}$ be of the form

$$
f(z)=z+\sum_{n=2}^{\infty} a_{n} z^{n}, g_{1}(z)=z+\sum_{n=2}^{\infty} c_{n} z^{n}, g_{2}(z)=z+\sum_{n=2}^{\infty} d_{n} z^{n},
$$

where $\left|a_{n}\right| \leq n,\left|c_{n}\right| \leq n,\left|d_{n}\right| \leq n, n=2,3, \ldots$. Then

$$
g_{1} * g_{2} \in T N_{\log 2}(f) .
$$

The number $\log 2$ is the best possible.

Proof. Since

$$
\left(g_{1} * g_{2}\right)(z)=z+\sum_{n=2}^{\infty} c_{n} d_{n} z^{n}
$$


then we have

The functions

$$
\sum_{n=2}^{\infty} \frac{1}{n^{2} 2^{n}}\left|c_{n} d_{n}-a_{n}\right| \leq \sum_{n=2}^{\infty} \frac{n^{2}+n}{n^{2} 2^{n}}=\log 2
$$

$$
f(z)=z-\sum_{n=2}^{\infty} n z^{n}, \quad g_{1}(z)=g_{2}(z)=z+\sum_{n=2}^{\infty} n z^{n}
$$

show that the number $\log \{2\}$ is the best possible. Therefore the proof is completed.

Definition $2.5([7])$. Let $A$ and $B$ be arbitrary subsets of the $\mathcal{A}$, and let $T$ be a sequence of positive number, then $\delta_{T}^{*}(A, B)$ is defined by

$$
\delta_{T}^{*}(A, B)=\inf \left\{\delta>0: B \subset T N_{\delta}(f) \text { for all } f \in A\right\} .
$$

Let us denote

$$
T(f, g)=\sum_{n=2}^{\infty} T_{n}\left|a_{n}-b_{n}\right|
$$

Therefore, we can write

$$
\begin{aligned}
\delta_{T}^{*}(A, B) & =\inf \{\delta: T(f, g)<\delta \text { for all } f \in A, g \in B\} \\
& =\sup \{T(f, g): f \in A, g \in B\},
\end{aligned}
$$

where the condition $T(f, g)<\delta$ means that the series $T(f, g)$ is convergent and its sum is less than $\delta$. Therefore, we see that $\delta_{T}^{*}(A, B)=\delta_{T}^{*}(B, A)$, and we will say that $\delta_{T}^{*}(A, B)$ is the $T$-factor with respect to the classes $A$ and $B$. Making use of the above definition, Corollary 2.2 and the consideration below Corollary 2.2, we can state next corollary where $T=\left\{T_{n}\right\}_{n=2}^{\infty}$ is again of the form (2.1).

Corollary 2.6. The $T$-factor with respect to the classes $\mathcal{S}$ and $\mathcal{S}$ satisfies the following inequality

$$
0.287 \cdots=\log \{4 / 3\} \leq \delta_{T}^{*}(\mathcal{S}, \mathcal{S}) \leq \log \{4 / e\}=0.386 \cdots .
$$

It is well known that the Koebe function and all its rotations belong to each of the classes $\mathcal{S}, \mathcal{S}^{*}$ and $\mathcal{K}$ (univalent, starlike and close-to-convex functions respectively), then Corollary 2.6 follows the next corollary.

Corollary 2.7. Let $A$ and $B$ be one of the classes $\mathcal{S}, \mathcal{S}^{*}$ or $\mathcal{K}$. Then

$$
\log \{4 / 3\} \leq \delta_{T}^{*}(A, B) \leq \log \{4 / e\} .
$$

In the same way as above, we can express Corollary 2.3 in terms $T$-factor. It is done in the next result.

Corollary 2.8. The $T$-factor with respect to the classes $\mathcal{C}$ of convex functions and $\mathcal{S}$ satisfies the following inequality

$$
0.24473 \cdots \leq \delta_{T}^{*}(\mathcal{C}, \mathcal{S}) \leq 0.275 \cdots
$$


Remark 2.9. Now we consider the "central" function with respect to coefficient in the class $\mathcal{C} o(1)$ which is denoted by $f_{c}(z)$ and defined by

$$
f_{c}(z)=\frac{1}{2}\left\{\frac{z}{1-z}+\frac{z}{(1-z)^{2}}\right\}=z+\sum_{n=1}^{\infty} \frac{n+1}{2} z^{n}, \quad|z|<1 .
$$

In [7] the authors showed that $f_{c} \in \mathcal{C} o(1)$.

Theorem 2.10. The following inclusion relation holds

$$
\mathcal{C} o(1) \subset T N_{\delta}\left(f_{c}\right),
$$

where $\delta=\log \sqrt{2 / e}+\pi^{2} / 24-(\log 2)^{2} / 4=0.13769 \cdots$.

Proof. Suppose that $f(z)=z+\sum_{n=2}^{\infty} a_{n} z^{n} \in \mathcal{C} o(1)$, then from (1.2), and using (2.3) and (2.4) with $x=-1 / 2$, we obtain

$$
\begin{aligned}
\sum_{n=2}^{\infty} T_{n}\left|a_{n}-\frac{n+1}{2}\right| & \leq \frac{1}{2} \sum_{n=2}^{\infty} \frac{n-1}{n^{2} 2^{n}} \\
& =\frac{1}{2}\left\{\log 2-\frac{1}{2}+f_{2}(1 / 2)-\frac{1}{2}\right\} \\
& =\frac{1}{2}\left\{\log 2-1+\frac{\pi^{2}}{12}-\frac{(\log 2)^{2}}{2}\right\} \\
& =0.13769 \cdots=\delta .
\end{aligned}
$$

Acknowledgment. The authors would like to express their sincerest thanks to the referees for a careful reading and various suggestions made for the improvement of the paper.

\section{References}

[1] F. G. Avkhadiev, Ch. Pommerenke, and K. J. Wirths, On the coefficients of concave univalent functions, Math. Nachr. 271 (2004), 3-9.

[2] Sharp inequalities for the coefficients of concave schlicht functions, Comment. Math. Helv. 81 (2006), no. 4, 801-807.

[3] F. G. Avkhadiev and K. J. Wirths, Convex holes produce lower bound for coefficients, Complex Var. Theory Appl. 47 (2002), no. 7, 553-563.

[4] U. Bednarz, Stability of the Hadamard product of $k$-uniformly convex and $k$-starlike functions in certain neighbourhood, Demonstratio Math. 38 (2005), no. 4, 837-845.

[5] U. Bednarz and S. Kanas, Stability of the integral convolution of $k$-uniformly convex and $k$-starlike functions, J. Appl. Anal. 10 (2004), no. 1, 105-115.

[6] U. Bednarz and J. Sokól, On the integral convolution of certain classes of analytic functions, Taiwanese J. Math. 13 (2009), no. 5, 1387-1396.

[7] _ T-neighborhoods of analytic functions, J. Math. Appl. 32 (2010), 25-32.

[8] A. Bielecki and Z. Lewandowski, Sur une généralisation de quelques théorèmes de $M$. Biernacki sur les fonctions analytiques, Ann. Polon. Math. 12 (1962), 65-70.

[9] P. L. Duren, Univalent functions, Springer Verlag, Grund. math. Wiss. 259, New York, Berlin, Heidelberg, Tokyo, 1983.

[10] R. Fournier, A note on neighbourhoods of univalent functions, Proc. Amer. Math. Soc. 87 (1983), no. $1,117-120$. 
[11] (1986), no. 20, 189-202.

[12] $ـ$ On neighbourhoods of univalent convex functions, Rocky Mountain J. Math. 16 (1986), no. 3, 579-589

[13] L. Lewin, Dilogarithms and Associated Functions, Macdonald, London, 1958.

[14] St. Ruscheweyh, Neighborhoods of univalent functions, Proc. Amer. Math. Soc. 81 (1981), no. 4, 521-527.

[15] S. Shams and S. R. Kulkarni, Certain properties of the class of univalent functions defined by Ruscheweyh derivative, Bull. Cal. Math. Soc. 97 (2005), no. 3, 223-234.

[16] T. Sheil-Small, On linear accessibility and the conformal mapping of convex domains, J. Analyse Math. 25 (1972), 259-276.

[17] T. Sheil-Small and E. M. Silvia, Neighborhoods of analytic functions, J. Analyse Math. 52 (1989), 210-240.

[18] J. Stankiewicz, Neighbourhoods of meromorphic functions and Hadamard products, Ann. Polon. Math. 46 (1985), 317-331.

SAEID SHAMs

Department of Mathematics

UNIVERSITY OF URMIA

URMIA, IRAN

E-mail address: s.shams@urmia.ac.ir

Ali EBADian

Department of Mathematics

PAyAmE NoOR University

TEHRAN, IRAN

E-mail address: a.ebadian@urmia.ac.ir

MaHTA SAYADiAZAR

Department of MATHEMATiCS

UNIVERSITY OF URMIA

URMIA, IRAN

E-mail address: m.sayyadiazar@yahoo.com

JANUSZ SOKÓE

Department of Mathematics

Rzeszów University of Technology

Al. Powstańców Warszawy 12, 35-959 Rzeszów, Poland

E-mail address: jsokol@prz.edu.pl 